\title{
Decoupled heat and charge rectification as many-body effect in quantum wires
}

\author{
Conor Stevenson and Bernd Braunecker \\ SUPA, School of Physics and Astronomy, University of St Andrews, North Haugh, St Andrews KY16 9SS, UK
}

(Dated: February 18, 2021)

\begin{abstract}
We show that for a quantum wire with a local asymmetric scattering potential the principal channels for charge and heat rectification decouple and renormalise differently under electron interactions, with heat rectification generally being more relevant. The polarisation of the rectification results from quantum interference and is tuneable through external gating. Furthermore, for spin polarised or helical electrons and sufficiently strong interactions a regime can be obtained in which heat transport is strongly rectified but charge rectification is very weak.
\end{abstract}

\section{INTRODUCTION}

Electronic technology relies significantly on the progressive miniaturisation of its components. This has started reaching into the quantum regime. It is thus natural to ask if genuine quantum effects can help to define new functionality, even if quantum computing itself is not targeted. This question is especially interesting when interactions are included, as indeed the latter become pertinent with the confinement of charges caused by the miniaturisation. Many-body correlations in particular can offer the opportunity to design properties not achievable through conventional electronics. In this paper we present such an example in which interactions are tuned to decouple charge and heat rectification.

Rectification, the diode effect, is characterised by an asymmetric current-voltage relation. In a conventional diode this asymmetry is introduced by $p$ and $n$ type doped sides of a semiconductor junction. Although the dopants create an electrostatic environment the resulting physics is understood on the single electron level. A many-body variant exists but relies on different physics. It was shown long ago that in quantum wires as illustrated in Fig. 1 a local scattering potential $U(x)$ causes a strong renormalisation of the current-voltage relation through electron interactions $[1,2]$. While the leading correction is independent of the potential's form, sub-leading orders are shape sensitive, and a spatially asymmetric potential induces rectification [3-5] which for strong interactions can become very large.

In this paper we investigate this scenario under the novel aspect of thermoelectric rectification where heat current is driven by a voltage $V$. In the nonlinear regime this is different from a temperature driven current which we do not consider. The thermoelectric response in quantum wires has been considered in various settings [6-12] but for rectification our focus is entirely on the heat flow from backscattering which to our knowledge has not been investigated before. The many-body setup is also different from the usual approaches to heat rectification that depend on an artful design of the system or the reservoirs [13-33]. With the tools of open quantum systems and quantum thermodynamics we derive an intuitive result that automatically incorporates the requirement of gauge invariance [26, 27, 34, 35] and evaluate (a)

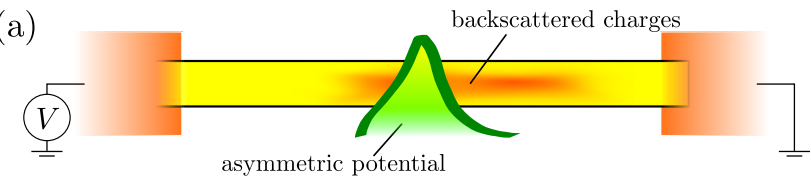

(b)

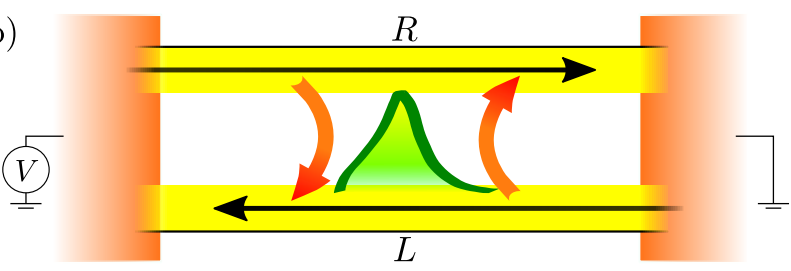

FIG. 1. (a) Scheme of the voltage $V$ driven quantum wire with a spatially asymmetric potential $U(x)$. Rectification arises from dressing $U(x)$ by backscattered charges together with renormalisation through interactions. (b) The wire as a thermodynamic system with right (left) moving modes $R(L)$ in equilibrium with the reservoir on their left (right). The reservoirs are fully absorbing for incoming particles. Backscattering by $U(x)$ (curved arrows) connects the $R$ and $L$ subsystems and causes the transport asymmetry under $V$.

it through nonequilibrium perturbation theory. Remarkably the asymmetry of the heat current appears already at the leading current renormalisation such that through interactions it decouples from charge rectification and generally dominates.

In addition to normal electrons we consider effectively spinless (e.g. polarised or helical) conductors. For the latter we find that for strong interactions the heat asymmetry can become as large as the heat current itself whereas the charge asymmetry remains very small. This produces the novel phenomenon of a conductor that acts as a heat diode but not as a charge diode. Furthermore whether the heat transport is reduced for positive or negative bias depends on quantum interference and can be switched even through small changes of the impurity potential which can be created through local external gates.

The plan of the paper is the following: In Sec. II we discuss the physics underlying the heat rectification. The model is introduced in Sec. III and quantitatively analysed for noninteracting electrons in Sec. IV. The modifications from interacting electrons is topic of Sec. $\mathrm{V}$ in which we highlight also how renormalisation effects cause a decoupling from charge rectification. Rectification effi- 
ciencies are investigated in Sec. VI before concluding in Sec. VII. The evaluation of the required correlation functions relies on standard techniques for one-dimensional conductors and a summary of the used bosonisation details is provided in Appendix A.

\section{PHYSICS BEHIND RECTIFICATION}

For a setup as in Fig. 1 the asymmetry causing rectification is due to the local potential $U(x)$ alone. In an interacting system backscattering on $U$, described by Fourier modes $U_{2 k_{F}}$ with $k_{F}$ the Fermi momentum, causes a strong renormalisation of transport $[1,2]$. But the usual leading correction, proportional to $\left|U_{2 k_{F}}\right|^{2}$, does not retain spatial information and does not contribute to rectification. A spatial asymmetry dependence, which then causes rectification, appears only at sub-leading orders with higher powers of $U$ in the renormalisation $[3,4]$ but for strong interactions they can grow in magnitude and indeed create a pronounced diode effect.

In contrast for energy or heat currents the dependence on the asymmetry of $U(x)$ appears remarkably already at leading order. The potential $U(x)$ contains two contributions, backscattering and forward scattering. The latter does not affect charge transport but changes locally the kinetic energy of the particles. This is conventionally captured by a local chemical potential $\mu(x)=\mu+U(x)$, which modifies the local energy density and enters linearly in the energy transfer by backscattering which remains proportional to $U^{2}$. We will show that the overall amplitude is $(U \star U)_{2 k_{F}} U_{2 k_{F}}^{*}$, with $\star$ the convolution of the Fourier modes. This amplitude is complex and through its phase retains the signature of the spatial asymmetry, thus taking the role occurring only at higher orders for charge rectification. Intuitively this process should be understood as that the electrons pick up a phase due to the locally modified density just before or after backscattering. This phase is different for counterflowing particles due to the asymmetry of $U(x)$. Since the backscattering process is local it convolves this phase of the incoming wave packets with the phase from the backscattering potential to the final momentum transfer $2 k_{F}$ required to change the direction of propagation of wave packets. The phases of the complex amplitudes are thus the result from the interference of the incoming with the outgoing wave packets and sensitive on the precise shape of $U(x)$. Due to the nonlocal nature of the Fourier transformation there is no intuitive way to anticipate the precise acquired phases but in Sec. IV and Fig. 2 we provide a concrete example illustrating how external gating can influence the interference to one's advantage.

The dependence of charge and heat current rectification causes then a decoupling of the corresponding renormalisation channels in an interacting system. This leads to different voltage $V$ dependences in the form of different power-law scalings. Since heat rectification arises at the most relevant order it usually dominates over charge rectification, and interactions can even be tuned such that heat rectification is strongly enhanced while charge rectification remains very small, both relative to their total currents. Such a device then operates as a good thermal diode without significant impact on charge rectification.

\section{MODEL AND CURRENTS}

For a quantitative evaluation we consider a onedimensional quantum wire connected to reservoirs on each end. For voltage $V$ driven thermoelectric transport the reservoirs do not have any specific form and their temperature is irrelevant as long as $V$ sets the dominant energy scale. The physics of a one-dimensional conductor is very susceptible to electron interactions, and the conventional Fermi liquid paradigm is generically replaced by the universality class of the Luttinger liquid [36]. The latter is characterised by collective charge and spin density modes which in part drastically change the shape of correlation functions in comparison with the Fermi liquid, and we will make use of this behaviour, in particular of the property that charge and heat rectification currents are differently renormalised. Since this physics is universal it can conveniently be accessed through an appropriately chosen model. We therefore describe the system in terms of the Tomonaga-Luttinger model [37$40]$ in which electron operators $\psi(x)$ are split into right $R$ and left $L$ moving modes with momenta close to $+k_{F}$ and $-k_{F}$. With $\psi_{R, L}(x)$ the corresponding field operators, the Hamiltonian without $U(x)$ becomes

$$
\begin{aligned}
H & =\int d x \sum_{\nu} \psi_{\nu}^{\dagger}(x)\left(\mu_{\nu}-\nu i \hbar v_{F} \partial_{x}\right) \psi_{\nu}(x) \\
& +\int d x d y \mathcal{V}(x-y) \psi^{\dagger}(x) \psi^{\dagger}(y) \psi(y) \psi(x),
\end{aligned}
$$

where $\nu=R, L=+,-$, the integration is over the wire length, $v_{F}$ is the Fermi velocity for the linearised dispersion, and $\mathcal{V}(x-y)$ the (screened) electron interaction. Spin is not written since all terms are spindiagonal but its influence is discussed later. As shown in Fig. 1 (a) the $R$ and $L$ movers have the chemical potentials $\mu_{R, L}$ that are set by the emitting reservoirs [3$6,41,42]$. The voltage drop is $V=\left(\mu_{R}-\mu_{L}\right) / e$, with $e$ the electron charge. For $\mu_{R} \neq \mu_{L}$ the Fermi momentum is adjusted to $k_{F}^{R, L}=k_{F}+\left(\mu_{R, L}-\mu_{0}\right) / \hbar v_{F}$, with $\mu_{0}$ the equilibrium chemical potential. The field operator is $\psi(x)=e^{i k_{F}^{R} x} \psi_{R}(x)+e^{-i k_{F}^{L} x} \psi_{L}(x)$. Without interactions the Hamiltonian decouples into $R$ and $L$ moving fermionic modes, providing the condition shown in Fig. 1 (b). Such a decoupling persists even with interactions if $\pi / k_{F}$ is not commensurate with the crystal lattice, and we exclude the latter special cases. The low energy eigenmodes become then collective density wave excitations that still are separate $R$ and $L$ movers, albeit both mixing the original $R$ and $L$ movers $[39,40]$. An appropriate 
proof of this decoupling can be given through the bosonisation technique which allows us in addition to evaluate all required correlators explicitly. This is a standard calculation which we use as a tool to supplement the results, but it is not of primary importance for the discussion otherwise. We therefore delegate the details to App. A and in the main discussion focus entirely on the resulting physics and its interpretation. Through the decoupling of modes we can thus always write $H=H_{L}+H_{R}$ with $H_{\nu}$ containing only $\nu$ moving eigenmodes. Scattering on $U(x)$ has the Hamiltonian

$$
H_{U}=\int d x \sum_{\nu, \nu^{\prime}} U(x) e^{-i\left(\nu k_{F}^{\nu}-\nu^{\prime} k_{F}^{\nu^{\prime}}\right) x} \psi_{\nu}^{\dagger}(x) \psi_{\nu^{\prime}}(x),
$$

where $U(x)$ is non-zero only in a small region $<\pi / k_{F}$ around $x=0$ and we assume that it is spatially asymmetric, $U(x) \neq U(-x)$. This potential takes two roles. For $\nu=\nu^{\prime}$ it describes forward scattering that can be added to $H_{\nu}$ by letting $\mu_{\nu}(x)=\mu_{\nu}+U(x)$. For $\nu \neq \nu^{\prime}$ the potential introduces backscattering between $R$ and $L$ movers, and we call this part of the Hamiltonian $H_{b}$. For a helical system (opposite spins bound to $R, L$ movers) $U$ is a magnetic impurity inducing both spin preserving forward and spin-flip backward scattering.

Backscattering is a relevant perturbation for electron transport $[1,2]$ but the leading term, proportional to $\left|U_{2 k_{F}}\right|^{2}$, is asymmetry insensitive. Thus charge rectification depends on sub-leading contributions $[3,4]$, revealed through the rectification particle current, $\dot{N}_{\nu}^{r}=$ $\dot{N}_{\nu}(V)+\dot{N}_{\nu}(-V)$, where $\dot{N}_{\nu}=\frac{d}{d t} N_{\nu}$ measures how particle numbers $N_{\nu}$ of $\nu$ movers change by backscattering. By particle conservation $\dot{N}_{R}=-\dot{N}_{L}$.

Identifying heat or energy transfer is a bit more subtle. We have to consider $R$ and $L$ movers as thermodynamic subsystems that are brought into contact through the interface Hamiltonian $H_{b}$ [see Fig. 1 (b)]. The energy flow into system $\nu$ is given by the change of the internal energy $E_{\nu}=\operatorname{Tr}_{\nu}\left\{H_{\nu} \rho_{\nu}\right\}$, with $\operatorname{Tr}_{\nu}$ the trace over the degrees of freedom of subsystem $\nu$ and $\rho_{\nu}$ the reduced density matrix obtained from the full density matrix $\rho$ through $\rho_{R, L}=\operatorname{Tr}_{L, R}\{\rho\}$. If we put all time dependence in $\rho$ and notice that we can write $E_{\nu}=\operatorname{Tr}\left\{H_{\nu} \rho\right\}$ with $\operatorname{Tr}$ the full trace we obtain

$$
\dot{E}_{\nu}=-\frac{i}{\hbar} \operatorname{Tr}\left\{H_{\nu}[H, \rho]\right\}=-\frac{i}{\hbar} \operatorname{Tr}\left\{\left[H_{\nu}, H_{b}\right] \rho\right\},
$$

where we have used the von Neumann equation for the time evolution of $\rho$, the cyclicity of the trace, and $\left[H_{\nu}, H_{\nu^{\prime}}\right]=0$. Notice that $\dot{E}_{R}=-\dot{E}_{L}$ although formally they differ by an interface term $\propto H_{b}$ [43]. But in steady state this term has expectation value zero. To identify the heat current $\dot{Q}_{\nu}$ through the interface $H_{b}$ we separate $\dot{E}_{\nu}$ into heat and work fluxes. The criterion for heat flux as the quantity that changes entropy [43, 44] would give $\dot{Q}_{\nu}=\dot{E}_{\nu}$ as $H_{b}$ mixes $R$ and $L$ states. But $H_{b}$ exchanges particles too such that for the grand canonical setting $\mu_{\nu} \dot{N}_{\nu}$ has to be split off from the heat flux and we obtain $\dot{Q}_{\nu}=\dot{E}_{\nu}-\mu_{\nu} \dot{N}_{\nu}$. This splitting reproduces the standard form of the change of thermodynamic potential $d E_{\nu}=d Q_{\nu}+\mu_{\nu} d N_{\nu}$ and is in particular necessary because it makes $\dot{Q}_{\nu}$ independent of the gauge fixing the origin of energy [26, 27, 34, 35]. Similarly to Eq. (3) we obtain

$$
\dot{Q}_{\nu}=-\frac{i}{\hbar} \operatorname{Tr}\left\{\left[H_{\nu}-\mu_{\nu} N_{\nu}, H_{b}\right] \rho\right\} .
$$

and the $\mu_{\nu} N_{\nu}$ term indeed cancels the $\mu_{\nu}$ in Eq. (1). The latter equation provides the earlier mentioned intuitive result for the backscattering induced heat current.

\section{NONINTERACTING ELECTRONS}

Remarkably heat rectification itself does not need interactions and arises as a clear quantum interference effect. Focusing on $\dot{Q}_{R}$ and using the fact that the $R, L$ decoupling of $H$ can be read off from the first line in Eq. (1), the standard anticommutation relations yield

$$
\begin{aligned}
& \dot{Q}_{R}=\frac{1}{\hbar} \int d x U(x) e^{i\left(k_{F}^{L}+k_{F}^{R}\right) x} \\
& \times \operatorname{Tr}\left\{\psi_{L}^{\dagger}(x)\left(U(x)-i \hbar v_{F} \partial_{x}\right) \psi_{R}(x) \rho\right\}+\text { c.c. }
\end{aligned}
$$

Although this result is derived for a noninteracting system we show in App. A that it remains unchanged for an interacting system. In Eq. (5) as well as in the interacting case below we can drop the $U$ independent term as it produces only a logarithmic correction to the amplitude and no rectification at the considered orders. Furthermore the $V$ dependence of $k_{F}^{\nu}$, in contrast to its role for $\dot{N}_{\nu}^{r}[3,4]$, just produces higher powers in $V$ and we set $k_{F}^{R}+k_{F}^{L} \approx 2 k_{F}$. The Keldysh nonequilibrium expansion of $\rho$ in $U$ gives at leading order

$$
\begin{aligned}
& \dot{Q}_{R}=\frac{-i}{\hbar^{2}} \int d x d x^{\prime} U^{2}(x) U\left(x^{\prime}\right) e^{i 2 k_{F}\left(x-x^{\prime}\right)} \int_{-\infty}^{0} d t \\
& \times\left\langle\left[\psi_{L}^{\dagger}(x, 0) \psi_{R}(x, 0), \psi_{R}^{\dagger}\left(x^{\prime}, t\right) \psi_{L}\left(x^{\prime}, t\right)\right]\right\rangle+\text { c.c. },
\end{aligned}
$$

where $\psi_{\nu}$ evolves under $H_{\nu}$ and the expectation value is over the uncoupled $R, L$ systems. Equation (6) describes the interference of an incoming wave packet with its backscattered counterpart. Due to the different powers $U^{2}(x)$ and $U\left(x^{\prime}\right)$ and the spatial asymmetry of $U$ this expression breaks the $L-R$ symmetry and thus the interference patterns are different for applied $\pm V$ voltages. To obtain a quantitative result for the interference we notice that $\psi_{\nu}(x, t)$ varies slowly on the scale $\pi / k_{F}$ which is much longer than the support of $U(x)$. This allows us to set the arguments $x, x^{\prime}$ of the field operators to 0 , and the spatial integration then provides the Fourier transforms $U_{k}$ of $U(x)$ and $\left(U^{2}\right)_{k}=(U \star U)_{k}$ of $U^{2}(x)$,

$$
\begin{aligned}
& \dot{Q}_{R}=\frac{-i}{\hbar^{2}}\left(U^{2}\right)_{2 k_{F}}^{*} U_{2 k_{F}} \int_{-\infty}^{0} d t \\
& \times\left\langle\left[\psi_{L}^{\dagger}(0,0) \psi_{R}(0,0), \psi_{R}^{\dagger}(0, t) \psi_{L}(0, t)\right]\right\rangle+\text { c.c. }
\end{aligned}
$$


The gauge transformation $\psi_{\nu}(x, t)=e^{-i \mu_{\nu} t / \hbar} \tilde{\psi}_{\nu}(x, t)$ sets the bulk $\mu_{R, L} \rightarrow 0$ but makes the $V$ dependence evident by giving rise to $e^{i\left(\mu_{R}-\mu_{L}\right) t / \hbar}=e^{i e V t / \hbar}$ in Eq. (7). If $e V$ is larger than the thermal energy we can neglect temperature for the evaluation of the correlators, which marks a difference to temperature driven transport [45]. The time dependence of the remaining correlators $\left\langle\tilde{\psi}_{\nu}^{\dagger}(0,0) \tilde{\psi}_{\nu}(0, t)\right\rangle$ and $\left\langle\tilde{\psi}_{\nu}(0,0) \tilde{\psi}_{\nu}^{\dagger}(0, t)\right\rangle$ is then $1 / t$, set by the cutoff of the energy integration by the Fermi surface [46]. By going to dimensionless variables $y=|e V| t / \hbar$ we see that $\dot{Q}_{R}$ scales as $|V|$. This linear response result is expected since Eq. (7) is identical to $\dot{N}_{R}$ except for the $U^{2}$ amplitude instead of $U$. If we collect all invariant parameters in the constant $C$ we obtain

$$
\dot{Q}_{R}=-\left(U^{2}\right)_{2 k_{F}}^{*} U_{2 k_{F}} C|e V| \int_{-\infty}^{0} d y \frac{i e^{\operatorname{sign}(V) i y}}{y^{2}}+\text { c.c. }
$$

The divergence at $y \rightarrow 0$ in the integral results from the constant density of states in the Tomonoga-Luttinger model and requires a cutoff by the true bandwidth. This cutoff could in principle produce a further $V$ dependence from the scaling $t \rightarrow y$ but the magnitude of currents is set by $V$ and has to vanish at $V=0$. Therefore the cutoff must drop out with the commutators in Eq. (7) and any singularity can be neglected in the evaluation of the integral. For $\dot{N}_{R}$ the first two factors in Eq. (8) would be $U_{2 k_{F}}^{*} U_{2 k_{F}}=\left|U_{2 k_{F}}\right|^{2}$ and the expression in front of the integral would be real. With the 'c.c' the integrand then becomes $\operatorname{sign}(V) 2 \sin (y) / y^{2}$ such that $\dot{N}_{R}$ just changes sign but not magnitude with $V \rightarrow-V$. Charge rectification thus requires higher order corrections [3, 4].

Heat current involves instead $\left(U^{2}\right)_{2 k_{F}}^{*} U_{2 k_{F}}$. For a real symmetric potential $U(x)=U(-x)$ the Fourier components are real, and rectification remains absent. But for a spatially asymmetric potential $\left(U^{2}\right)_{2 k_{F}}^{*} U_{2 k_{F}}=$ $\left|\left(U^{2}\right)_{2 k_{F}} U_{2 k_{F}}\right| e^{i \alpha}$ is complex with a nonzero phase $\alpha$. The integrand becomes $2[\operatorname{sign}(V) \cos (\alpha) \sin (y)+$ $\sin (\alpha) \cos (y)] / y^{2}$. The term in $\sin (\alpha)$ is invariant under the sign of $V$ showing that heat current rectification exists even for a noninteracting system. If we define $\dot{Q}_{\nu}^{r}=\dot{Q}_{\nu}(V)+\dot{Q}_{\nu}(-V)$ as the rectification heat current measuring the asymmetry between $\pm V$ bias, we have thus found that

$$
\dot{Q}_{R}^{r}=\sin (\alpha)|V|\left|\left(U^{2}\right)_{2 k_{F}} U_{2 k_{F}}\right| C^{\prime},
$$

where the constant $C^{\prime}$ absorbs $C$ and the value of the remaining integration. An identical result holds for $\dot{Q}_{L}^{r}$ with $R \rightarrow L$ and $\alpha \rightarrow-\alpha$.

The phase $\alpha$ therefore captures the quantum interference resulting from Eq. (6) and is thus very sensitive to the precise shape of $U(x)$, such that generally the sign of $\sin (\alpha)$ is arbitrary. But this sensitivity allows also tuning for which only slight changes of $U(x)$ are required. In Fig. 2 we provide an example for $U(x)$ being the sum of two Lorentzians, and show that even a change of amplitude by just a few percent can completely reverse the

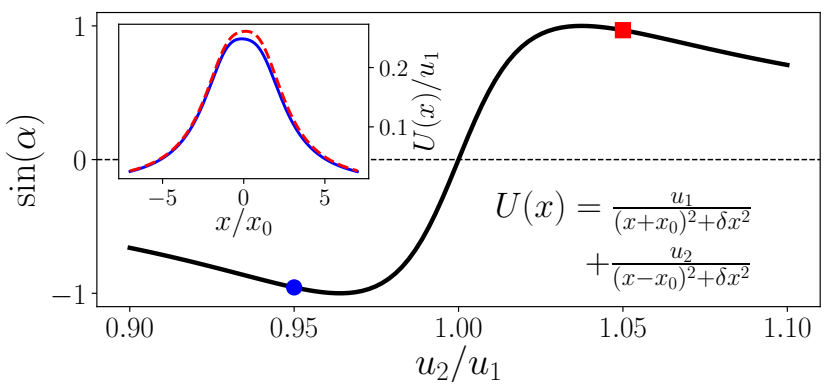

FIG. 2. Sensitivity of $\sin (\alpha)$ in Eq. (9) to the shape of $U(x)$, given here by the sum of two Lorentzians as indicated in the figure with parameters $\delta x=2 x_{0}$ and $k_{F}=0.4 / x_{0}$ in generic units $x_{0}, u_{1}$. The inset shows $U(x)$ for the ratios $u_{2} / u_{1}$ marked by the circle (solid line) and square (dashed line).

polarity. This sensitivity is a general feature of the asymmetry but independent of the shape of $U(x)$ otherwise. Asymmetric potentials are naturally realised when impurities appear close together within a Fermi wavelength [3-5]. Nearby narrow gates could then ensure sufficient tuneability. With typical Fermi wavelengths around 100 nm a direct creation by state-of-the-art gates could also be considered.

\section{INTERACTING ELECTRONS}

Interactions cause a significant renormalisation of the backscattering amplitude and hence of the rectification properties. This renormalisation occurs because backscattering locally changes the charge density in the vicinity of $U(x)$, so that an incoming wave packet experiences the combined effect from the potential $U(x)$ and the interaction with the displaced charges. This causes a self-consistent dressing of the potential and, for repulsive scatterers, a strong enhancement of the effective backscattering amplitude $[1,2]$. Underlying to this strong response is that in one dimension interactions destabilise the Fermi liquid and cause an instability towards density fluctuations. The universality class describing this physics is the Luttinger liquid [36, 39, 40], and the bosonisation method provides for the latter a standard technique to compute correlation functions at arbitrary interaction strength. For the present discussion our focus is on the effect of the results obtained through this technique, and we provide thus the necessary details on how to obtain the results in App. A. The correlators in Eq. (7) are then modified from $1 / t^{2}$ to $1 / t^{\gamma}[39,40]$ where $\gamma=2 K$ for the spinless and $\gamma=K_{c}+K_{s}$ for the spinful case. The parameters $K$ and $K_{c, s}$ capture all interactions. $K, K_{c}=1$ is the noninteracting case, $0<K, K_{c}<1$ encodes repulsive and $K, K_{c}>1$ attractive interactions. For the spinful case, if spin $\mathrm{SU}(2)$ symmetry is preserved $K_{s}=1$ and broken $K_{s}>1$. We exclude $K_{s}<1$ as it would represent an instability to spin density waves and require specially tuned spin interactions. 


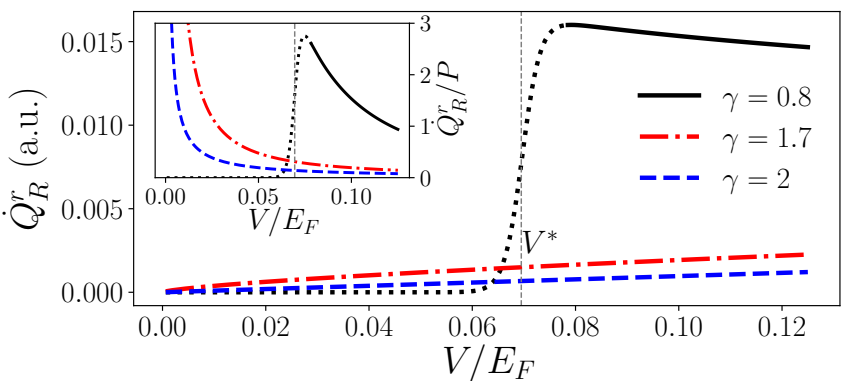

FIG. 3. Heat rectification as function of voltage for $U$ corresponding to the red square in Fig. 2 (with $u_{1} / E_{F}=0.7$ and $E_{F}$ setting the order of the bandwidth). Interactions with $\gamma<2$ enhance the noninteracting $\gamma=2$. For $\gamma<1$ (possible only for spin polarised electrons) a maximum enhancement is reached near $V=V^{*}$ where Eq. (9) crosses over to strong coupling scaling and $\dot{Q}_{R}^{r}$ decreases again to zero (expected trend by dotted line). The inset shows the corresponding efficiency $\dot{Q}_{R}^{r} / P$, with $P$ the dissipated power. While the scaling is exact only the order of magnitude is known for the amplitudes and we have set $C^{\prime}=1$.

The decoupling into $R$ and $L$ moving eigenmodes persists, and although the eigenmodes turn into renormalised density waves we show in App. A that Eq. (7) remains valid. The evaluation of the correlators is identical to those for the backscattering current [1] and changes the voltage dependence in Eq. (9) from $|V|$ to

$$
\dot{Q}_{\nu}^{r} \sim|V|^{\gamma-1}
$$

Since for repulsive interactions $\gamma<2$ this boosts the rectification current. In comparison charge rectification $\dot{N}_{\nu}^{r}=\dot{N}_{\nu}(V)+\dot{N}_{\nu}(-V)$ scales with $|V|^{\gamma_{c}}$ where $\gamma_{c}=\min (2 K, 6 K-2)$ for spinless [3] and $\gamma_{c}=\min \left(K_{c}+\right.$ $\left.K_{s}, 4 K_{c}, 3 K_{c}+K_{s}-2,12 K_{c}-2\right)$ for spinful electrons [4]. Heat and charge rectification thus decouple, and since $\dot{Q}_{\nu}^{r}$ arises from higher relevant contributions it is usually more significant.

Notice that these currents are obtained perturbatively on top of the heat or charge transfer between the reservoirs which from standard transport theory are proportional to $V$. Particularly interesting is when $\gamma-1$ or $\gamma_{c}$ becomes negative. Then the current increases when lowering $V$ until at some $V^{*}$ it becomes as large as the unperturbed current $\propto V$. Perturbation theory must then be replaced by a strong coupling calculation. Since currents must vanish at $V=0$ the currents decrease then again. Near $V^{*}$ backscattering and thus rectification are largest. Since $K_{s} \geq 1$ the spinful case has never $\gamma<1$ but this can be achieved for spin polarised electrons when $K<1 / 2$. If furthermore $K>1 / 3$ then charge rectification keeps $\gamma_{c}>0$ [3], making the decoupling of heat and charge rectification most pronounced, with strongly rectified heat and only weakly asymmetric charge current. Figure 3 shows $\dot{Q}_{R}^{r}$ for different $\gamma$. For $\gamma<1$ we interpolate to the strong coupling scaling $\dot{Q}_{R}^{r} \sim|V|^{4 / \gamma-1}[1]$ across $V^{*}$.

\section{RECTIFICATION EFFICIENCY}

For a good diode the ratio $r=\dot{Q}_{R}(-V) / \dot{Q}_{R}(V)$ is either $r \ll 1$ or $r \gg 1$. In the Tomonaga-Luttinger model an exact calculation of $r$ is tricky due to the required cutoffs. But Eq. (8) shows that

$$
r=\frac{A \sin (\alpha)-B \cos (\alpha)}{A \sin (\alpha)+B \cos (\alpha)}
$$

where $A$ and $B$ are of the same order. Therefore $r$ is tuneable through $\alpha$ to any value. Although its initial value is arbitrary this provides the advantage by tuning through gates. In Sec. IV we indeed have highlighted the sensitivity of $\alpha$ to small changes of gating bias such that the nonuniversality of $r$ can be used to turn the system into an actively programmable heat diode.

The efficiency of the heat transport is assessed by comparing $\dot{Q}_{R}^{r}$ to the total dissipated power $P=I V$ (Joule heating). Since the total current $I \propto V$ we obtain $\dot{Q}_{R}^{r} / P \sim|V|^{\gamma-3}$. For $1<\gamma<2$ the divergence at $V \rightarrow 0$ tells that heat rectification is most effective when dissipation is generally low. For $\gamma<1$ there is a strong suppression at $V<V^{*}$ and the benefit of strong rectification near $V^{*}$ involves a larger dissipation. This behaviour is illustrated in the inset of Fig. 3. Notice that since temperature of the reservoirs does not appear in these considerations there is no counterpart of the thermoelectric figure of merit $Z T$ and we use $\dot{Q}_{R}^{r} / P$ instead.

\section{CONCLUSIONS}

We have investigated how asymmetric potentials cause heat current rectification in quantum wires. Although the effect appears already for noninteracting particles through interference of the backscattered wave packets, it becomes most interesting in an interacting system. For the latter the charge and heat rectification decouple and are characterised by a different voltage dependence. The decoupling becomes more pronounced with increasing interactions, and in particular for spin polarised electrons at interaction strengths $1 / 3<K<1 / 2$ we predict a strong effect in which the heat rectification is strong but charge rectification remains weak. Such interaction strengths are not untypical for high quality conductors. For instance, GaAs quantum wires can be tuned to $K_{c} \sim 0.4[47,48]$ and are candidates for a helical transition [49] that would provide the further reduction of the spin degree of freedom. We have furthermore discussed that the rectification polarity is easily manipulatable through local gating. The basis of this is the sensitive dependence of the quantum interference amplitude on the detailed shape of the backscattering potential. This sensitivity makes the amplitude nonuniversal, which is quite common for one-dimensional systems, but it makes it thus also very suitable for an easy tuning of the polarisation of the rectifier. We have indeed shown 
that changes of a few percent of the asymmetric shape of the potential can be sufficient for a full polarisation reversal. This can be achieved through local gating and hence could make such a system useful as a programmable heat rectifier.

\section{ACKNOWLEDGMENTS}

We thank P. Jacquod for a discussion that has strongly inspired this work, and we thank D. E. Feldman, P. Jacquod, J. B. Marston, and Z. Zhuang for helpful comments. C.S. acknowledges the support by the EPSRC under Grant No. EP/N509759/1. The work presented in this paper is theoretical. No data has been produced and supporting research data is not required.

\section{Appendix A: Energy currents for interacting systems in bosonisation formalism}

The analysis of backscattering induced heat rectification above relies on the treatment of $R$ and $L$ moving modes as two distinguishable transport channels. The decoupling of these two channels appears naturally for noninteracting systems but becomes more subtle when interactions are involved. Indeed the general form of the interaction Hamiltonian

$$
H_{\mathrm{int}}=\int d x d y \mathcal{V}(x-y) \psi^{\dagger}(x) \psi^{\dagger}(y) \psi(y) \psi(x),
$$

with $\psi(x)=e^{i k_{F}^{R} x} \psi_{R}(x)+e^{-i k_{F}^{L} x} \psi_{L}(x)$, clearly couples $R$ and $L$ movers. However, as long as the fermion density is not commensurate with the underlying lattice most of the terms in $H_{\text {int }}$ are irrelevant in the renormalisation group sense [39, 40], and the only remaining interactions are of the form

$H_{\mathrm{int}}=\sum_{\nu, \nu^{\prime}=R, L} \int d x d y \mathcal{V}(x-y) \psi_{\nu}^{\dagger}(x) \psi_{\nu^{\prime}}^{\dagger}(y) \psi_{\nu^{\prime}}(y) \psi_{\nu}(x)$.

Within the Luttinger liquid paradigm (see [39, 40] for full details on the formalism used in this appendix) the fermionic Hamiltonian is then mapped onto a set of bosonic, harmonic oscillator type Hamiltonians, with boson fields representing the density fluctuations of the $R$ and $L$ movers. The interactions in Eq. (A2) cause a coupling between the $R$ and $L$ type boson fields for $\nu \neq \nu^{\prime}$, but this coupling remains bilinear so that the Hamiltonian is a quadratic form described by a $2 \times 2$ matrix for the $R, L$ fields which can be straightforwardly diagonalised. Although they mix contributions from both the original $R$ and $L$ movers, the resulting eigenmodes $\phi_{R, L}$ describe wave packets that move only to the right or the left, and hence maintain effectively the decoupling of $R$ and $L$ moving modes. The resulting Hamiltonians can be written for the spinless (or spin polarised) case as

$$
H_{\nu}=\int d x \frac{v}{4 \pi K}\left(\partial_{x} \phi_{\nu}(x)\right)^{2}+\mu_{\nu} N_{\nu},
$$

for $\nu=R, L$, where the fields obey the commutation relations

$$
\left[\phi_{\nu}\left(x^{\prime}\right), \partial_{x} \phi_{\nu^{\prime}}(x)\right]=i \pi K \delta_{\nu, \nu^{\prime}} \delta\left(x-x^{\prime}\right),
$$

i.e. $\phi_{\nu}$ and $\partial_{x} \phi_{\nu}$ are conjugate boson fields up to a normalisation. The parameter $K$ results from the diagonalisation of the $2 \times 2$ matrix and thus encodes the entire effect of $H_{\text {int }}$. It takes the values discussed in Sec. V.

The term $\mu_{\nu} N_{\nu}$ in Eq. (A3) contains the energy correction from the chemical potentials $\mu_{\nu}$ and the particle numbers $N_{\nu}$ of $\nu$ movers. This term depends on the choice of gauge for $\mu_{\nu}$ but drops out in the gauge independent expressions considered below and for the heat currents $\dot{Q}_{\nu}$ discussed in the main text.

The original fermion operators are expressed in terms of these eigenmodes as

$$
\psi_{\nu}(x)=\frac{\eta_{\nu}}{\sqrt{2 \pi a}} e^{-\frac{i}{2}\left(\nu-K^{-1}\right) \phi_{L}(x)-\frac{i}{2}\left(\nu+K^{-1}\right) \phi_{R}(x)},
$$

with the signs $\nu=R=+$ and $\nu=L=-$, and $a$ a short distance cutoff, typically on the order of the lattice spacing. The $\eta_{\nu}$ are operators that lower overall fermion number by one and guarantee the fermionic exchange statistics. For the further analysis they do not play any further role and can be dropped henceforth.

The forward scattering term on the impurity is obtained from point splitting of the densities,

$$
\psi_{\nu}^{\dagger}(x) \psi_{\nu}(x)=\frac{\nu+K^{-1}}{2 \pi} \partial_{x} \phi_{R}(x)+\frac{\nu-K^{-1}}{2 \pi} \partial_{x} \phi_{L}(x) .
$$

We have omitted here a term proportional to the average particle density $k_{F, \nu}$ as it contributes only a constant to the Hamiltonian. Consequently we have

$$
U(x) \sum_{\nu} \psi_{\nu}^{\dagger}(x) \psi_{\nu}(x)=\frac{U(x)}{\pi K}\left(\partial_{x} \phi_{R}(x)-\partial_{x} \phi_{L}(x)\right) .
$$

This term separates thus well into $R$ and $L$ moving contributions such that the total $\nu$ moving Hamiltonian after subtraction of the offset by the chemical potential reads

$$
H_{\nu}-\mu_{\nu} N_{\nu}=\int d x\left[\frac{v}{4 \pi K}\left(\partial_{x} \phi_{\nu}(x)\right)^{2}+\nu \frac{U(x)}{\pi K} \partial_{x} \phi_{\nu}(x)\right] .
$$

On the other hand, the backscattering Hamiltonian $H_{b}$ becomes

$$
\begin{aligned}
H_{b} & =\int d x U(x) e^{-2 i k_{F} x} \psi_{R}^{\dagger}(x) \psi_{L}(x)+\text { h.c. } \\
& =\int d x \frac{U(x)}{2 \pi a} e^{-2 i k_{F} x} e^{i \phi_{L}(x)+i \phi_{R}(x)}+\text { h.c. }
\end{aligned}
$$


with $2 k_{F}=k_{F}^{R}+k_{F}^{L}$. The interaction caused renormalisation causes further effective multi-particle backscattering terms in $H_{b}[1,2]$. For the main correction to the current and thus the main contribution to $\dot{Q}_{\nu}^{r}$ these are less relevant though and do not need to be considered. Charge rectification on the other hand depends directly on these multi-particle terms which were accordingly analysed in detail in Refs. [3-5]. Hence the cited different scaling laws for charge rectification.

For the heat transferred through backscattering, expressed by the commutator $\left[H_{\nu}-\mu_{\nu} N_{\nu}, H_{b}\right]$ we therefore need to evaluate the commutators of $\left(\partial_{x} \phi_{\nu}\right)^{2}$ and $\partial_{x} \phi_{\nu}$ with $H_{b}$ as given in Eq. (A9). From the commutation relations (A4) we see that

$$
\left[\partial_{x} \phi_{\nu}(x), e^{i \phi_{L}\left(x^{\prime}\right)+i \phi_{R}\left(x^{\prime}\right)}\right]=\pi K e^{i \phi_{L}(x)+i \phi_{R}(x)} \delta\left(x-x^{\prime}\right)
$$

The commutator with $\left(\partial_{x} \phi_{\nu}\right)^{2}$ takes a similar form with an additional factor $\partial_{x} \phi_{\nu}$. As noted the main text such terms produce less relevant logarithmic corrections to the leading expression so that we can leave them aside. The commutator for the heat backscattering current is then given by

$$
\begin{aligned}
& {\left[H_{\nu}-\mu_{\nu} N_{\nu}, H_{b}\right]} \\
& =\nu \int d x U^{2}(x) \frac{e^{-2 i k_{F} x}}{2 \pi a} e^{i \phi_{L}(x)+i \phi_{R}(x)}-\text { h.c. } \\
& =\nu \int d x U^{2}(x) e^{-2 i k_{F} x} \psi_{R}^{\dagger}(x) \psi_{L}(x)-\text { h.c. }
\end{aligned}
$$

This is exactly the same result obtained from the pure fermionic description of Eq. (5) but obtained here for a general interacting system with arbitrary values of $K$. It is notable that the backscattering heat current operator has no direct $K$ dependence.

When the spin degree of freedom is taken into account the bosonic fields double into charge and spin fluctuations but the structure of the equations and the identities are up to extra charge and spin labels identical. Equation (A11) is again unchanged from its noninteracting fermionic expression.

Finally the evaluation of the correlation functions in Eq. (7) follows the standard method. The expectation values in Eq. (7) can be reduced to the computation of bosonic correlators through the identity $\left\langle e^{i \phi_{\nu}(t)} e^{-i \phi_{\nu}(0)}\right\rangle=e^{\left\langle\phi_{\nu}(t) \phi_{\nu}(0)-\left[\phi_{\nu}^{2}(t)+\phi_{\nu}^{2}(0)\right] / 2\right\rangle}$ which is valid for a quadratic bosonic theory. Here we have set $\phi_{\nu}(t)=\phi_{\nu}(x=0, t)$. Focusing again on the spinless case the bosonic correlators are then evaluated as [39, 40]

$$
\left\langle\phi_{\nu}(t) \phi_{\nu}(0)-\left[\phi_{\nu}^{2}(t)+\phi_{\nu}^{2}(0)\right] / 2\right\rangle=-K \ln [(i a-v t) / i a],
$$

where $a$ is the short distance cutoff of the theory and $v=v_{F} / K$ the interaction renormalised Fermi velocity. The correlators in Eq. (7) lead to the exponential of two such bosonic correlators which thus provides the time dependence $1 / t^{\gamma}$ with $\gamma=2 K$ as discussed in Sec. V. The limit $K=1$ matches indeed the noninteracting case.

For the spinful case the eigenmodes $\phi_{\lambda, \nu}$ acquire the further index $\lambda=c, s$ expressing the charge and spin degrees of freedom. The latter are independent and obey the same commutation relations (A4) with an additional $\delta_{\lambda, \lambda^{\prime}}$ factor. The Hamiltonian decomposes into four terms $H_{\lambda, \nu}$ each of which is of the form of Eq. (A3) with the replacement $K \rightarrow K_{\lambda}$. The same replacement of $K$ is made for the correlators in Eq. (A12). For fermion operators the exponential in Eq. (A5) is replaced by $\psi_{\nu, \sigma} \sim e^{-i 2^{-3 / 2}\left(\varphi_{c, \nu}+\sigma \varphi_{s, \nu}\right)}$ where $\sigma= \pm$ is the additional spin index and $\varphi_{\lambda, \nu}=\left(\nu-K_{\lambda}^{-1}\right) \phi_{\lambda, L}+\left(\nu+K_{\lambda}^{-1}\right) \phi_{\lambda, R}$. Consequently the exponent $\gamma$ for the time dependence in the heat current correlators is replaced by $\gamma=K_{c}+K_{s}$ as described in Sec. V.
[1] C. L. Kane and M. P. A. Fisher, Phys. Rev. B 46, 15233 (1992).

[2] A. Furusaki and N. Nagaosa, Phys. Rev. B 47, 4631 (1993).

[3] D. E. Feldman, S. Scheidl, and V. M. Vinokur, Phys. Rev. Lett. 94, 186809 (2005).

[4] B. Braunecker, D. E. Feldman, and J. B. Marston, Phys. Rev. B 72, 125311 (2005).

[5] B. Braunecker, D. E. Feldman, and F. Li, Phys. Rev. B 76, 085119 (2007).

[6] I. V. Krive, I. A. Romanovsky, E. N. Bogachek, A. G. Scherbakov, and U. Landman, Low Temp. Phys. 27, 821 (2001).

[7] M. R. Li and E. Orignac, Europhys. Lett. 60, 432 (2002).

[8] A. Garg, D. Rasch, E. Shimshoni, and A. Rosch, Phys. Rev. Lett. 103, 096402 (2009).

[9] N. Wakeham, A. F. Bangura, X. Xu, J. F. Mercure, M. Greenblatt, and N. E. Hussey, Nat. Commun. 2, 396 (2011).
[10] W. DeGottardi and K. A. Matveev, Phys. Rev. Lett. 114, 236405 (2015).

[11] D. S. Shapiro, D. E. Feldman, A. D. Mirlin, and A. Shnirman, Phys. Rev. B 95, 195425 (2017).

[12] Y. V. Ivanov and O. N. Uryupin, Semic. 53, 641 (2019).

[13] B. Li, L. Wang, and G. Casati, Phys. Rev. Lett. 93, 184301 (2004).

[14] C. W. Chang, D. Okawa, A. Majumdar, and A. Zettl, Science 314, 1121 (2006).

[15] D. Segal, Phys. Rev. Lett. 100, 105901 (2008).

[16] R. Scheibner, M. König, D. Reuter, A. D. Wieck, C. Gould, H. Buhmann, and L. W. Molenkamp, New J. Phys. 10, 083016 (2008).

[17] F. Zhan, N. Li, S. Kohler, and P. Hänggi, Phys. Rev. E 80, 061115 (2009).

[18] L.-A. Wu, C. X. Yu, and D. Segal, Phys. Rev. E 80, 041103 (2009).

[19] W. Kobayashi, Y. Teraoka, and I. Terasaki, Appl. Phys. Lett. 95, 171905 (2009). 
[20] D. M.-T. Kuo and Y.-c. Chang, Phys. Rev. B 81, 205321 (2010).

[21] N. A. Roberts and D. G. Walker, Int. J. Therm. Sci. 50, 648 (2011).

[22] H. Tian, D. Xie, Y. Yang, T. L. Ren, G. Zhang, Y. F. Wang, C. J. Zhou, P. G. Peng, L. G. Wang, and L. T. Liu, Sci. Rep. 2, 523 (2012).

[23] J. Ren and J.-X. Zhu, Phys. Rev. B 87, 241412(R) (2013).

[24] F. Giazotto and F. S. Bergeret, Appl. Phys. Lett. 103, 242602 (2013).

[25] M. J. Martínez-Pérez and F. Giazotto, Appl. Phys. Lett. 102, 182602 (2013).

[26] J. Meair and P. Jacquod, J. Phys. Cond. Mat. 25, 082201 (2013).

[27] D. Sánchez and R. López, Phys. Rev. Lett. 110, 026804 (2013).

[28] A. Fornieri, M. J. Martínez-Pérez, and F. Giazotto, Appl. Phys. Lett. 104, 183108 (2014).

[29] J. Jing, D. Segal, B. Li, and L.-A. Wu, Sci. Rep. 5, 15332 (2015).

[30] M. J. Martínez-Pérez, A. Fornieri, and F. Giazotto, Nat. Nanotechnol. 10, 303 (2015).

[31] K. Joulain, J. Drevillon, Y. Ezzahri, and J. OrdonezMiranda, Phys. Rev. Lett. 116, 200601 (2016).

[32] J. Ordonez-Miranda, Y. Ezzahri, and K. Joulain, Phys. Rev. E 95, 022128 (2017).

[33] R. Nakai and N. Nagaosa, Phys. Rev. B 99, 115201 (2019).

[34] T. Christen and M. Büttiker, Europhys. Lett. 35, 523 (1996).

[35] R. S. Whitney, Phys. Rev. B 87, 115404 (2013).
[36] F. D. Haldane, J. Phys. C Solid State Phys. 14, 2585 (1981).

[37] S.-i. Tomonaga, Prog. Theor. Phys. 5, 544 (1950).

[38] J. M. Luttinger, J. Math. Phys. 4, 1154 (1963).

[39] A. O. Gogolin, A. A. Nersesyan, and A. M. Tsvelik, Bosonization and strongly correlated systems (Cambridge University Press, 1998).

[40] T. Giamarchi, Quantum Physics in One Dimension (Oxford University Press, 2007).

[41] R. Egger and H. Grabert, Phys. Rev. B 58, 10761 (1998).

[42] M. P. A. Fisher and L. I. Glazman, in Mesoscopic Electron Transp. (Springer Netherlands, 1997) pp. 331-373.

[43] H. Hossein-Nejad, E. J. O'Reilly, and A. Olaya-Castro, New J. Phys. 17, 075014 (2015).

[44] H. Weimer, M. J. Henrich, F. Rempp, H. Schröder, and G. Mahler, EPL 83, 30008 (2008).

[45] C. L. Kane and M. P. Fisher, Phys. Rev. Lett. 76, 3192 (1996).

[46] P. Nozières and C. T. De Dominicis, Phys. Rev. 178, 1097 (1969).

[47] O. M. Auslaender, A. Yacoby, R. De Picciotto, K. W. Baldwin, L. N. Pfeiffer, and K. W. West, Science 295, 825 (2002).

[48] H. Steinberg, G. Barak, A. Yacoby, L. N. Pfeiffer, K. W. West, B. I. Halperin, and K. Le Hur, Nat. Phys. 4, 116 (2008).

[49] C. P. Scheller, T. M. Liu, G. Barak, A. Yacoby, L. N. Pfeiffer, K. W. West, and D. M. Zumbühl, Phys. Rev. Lett. 112, 066801 (2014). 